\title{
Erratum to: Elevated Cerebral Cortical CD24 Levels in Patients and Mice with Traumatic Brain Injury: A Potential Negative Role in Nuclear Factor Kappa B/Inflammatory Factor Pathway
}

\author{
Wei Li • Hai-Ping Ling • Wan-Chun You • \\ Huan-Dong Liu • Qing Sun • Meng-Liang Zhou • \\ Wei Shen · Jin-Bing Zhao • Lin Zhu • Chun-Hua Hang
}

Published online: 11 January 2015

(C) Springer Science+Business Media New York 2015

Erratum to: Mol Neurobiol (2014): pp 187-198

DOI 10.1007/s12035-013-8509-4

The original paper of this article unfortunately contains an error.

In Fig. 5, the sham CD241/Iba1 group (Fig. 5q-t), the authors showed the wrong sample fields of TBI group rather than sham group.

The authors regret this error and hereby publishing the corrected Fig 5.

The online version of the original article can be found at http://dx.doi.org/ 10.1007/s12035-013-8509-4.

W. Li • W.-C. You • Q. Sun • M.-L. Zhou • L. Zhu • C.-H. Hang ( $\bowtie)$ Department of Neurosurgery, Jinling Hospital, School of Medicine, Nanjing University, 305 East Zhongshan Road,

Nanjing 210002, Jiangsu Province, People's Republic of China

e-mail: hang_neurosurgery@163.com

H.-P. Ling $\cdot$ H.-D. Liu $・$ M.-L. Zhou $\cdot$ W. Shen $\cdot$ L. Zhu

Department of Neurosurgery, Jinling Hospital, School of Medicine, Southern Medical University (Guangzhou), 305 East Zhongshan Road, Nanjing 210002, Jiangsu Province, People's Republic of China

J.-B. Zhao

Department of Neurosurgery, Nanjing Brain Hospital, Nanjing Medical University, 264 Guangzhou Road, Nanjing 210029, Jiangsu

Province, People's Republic of China 


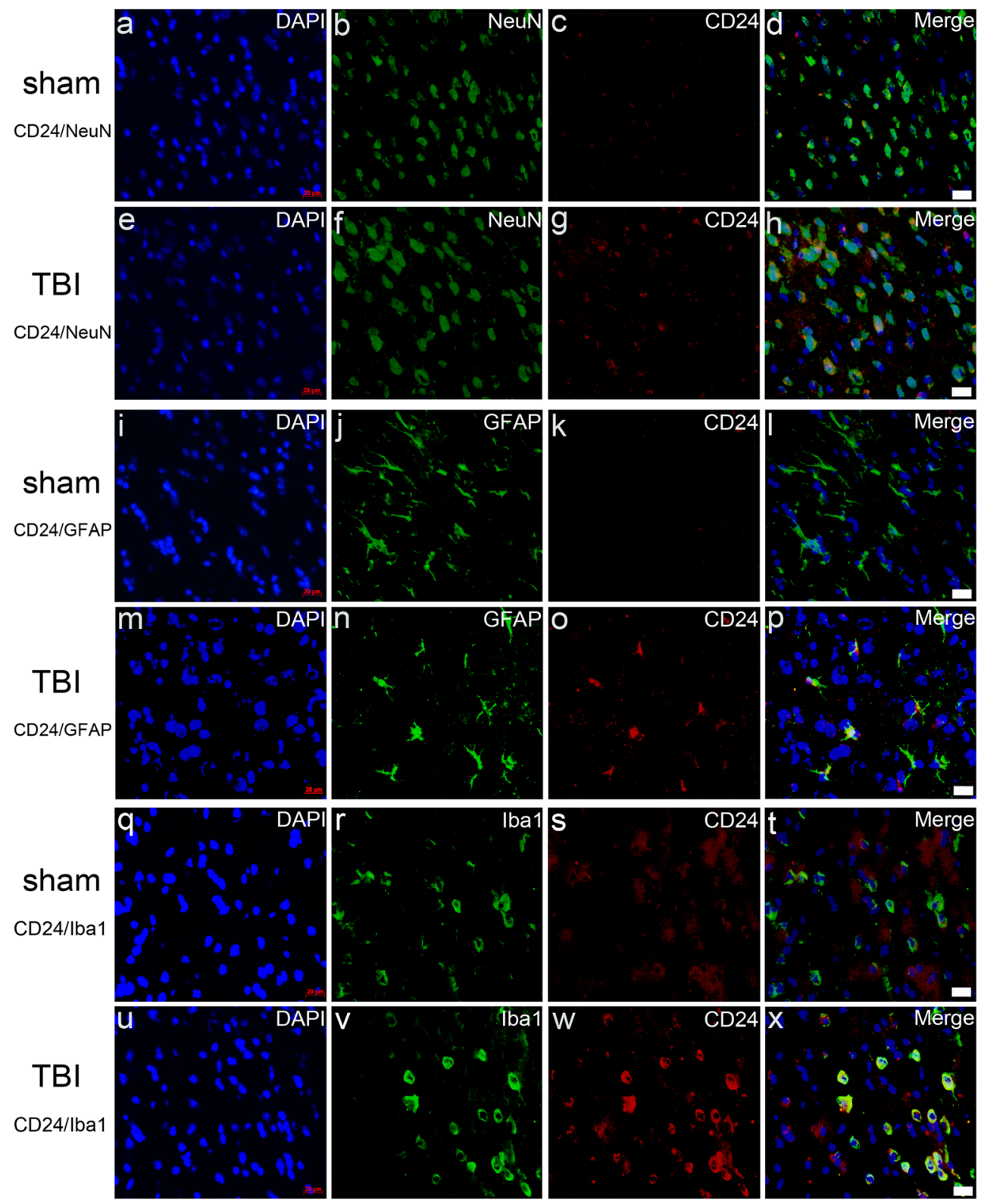

Fig. 5 CD24 expression and cellular distribution were detected by double immunohistofluorescent staining in mice. $\mathrm{CD} 24 / \mathrm{NeuN}$ double staining in the sham group (a-d) and TBI group on day $7(\mathbf{e}-\mathbf{h})(\mathrm{CD} 24=r e d, \mathrm{NeuN}=$ green, $\mathrm{DAPI}=$ blue); $\mathrm{CD} 24 / \mathrm{GFAP}$ double staining in the sham group (i-l) and TBI group on day $7(\mathbf{m}-\mathbf{p})(\mathrm{CD} 24=$ red, GFAP $=$ green, $\mathrm{DAPI}=$ blue); $\mathrm{CD} 24 / \mathrm{Iba} 1$ double staining in the sham group $(\mathbf{q}-\mathbf{t})$ and TBI group on day $7(\mathbf{u}-\mathbf{x})(\mathrm{CD} 24=r e d$, Iba1 $=$ green, DAPI=blue); CD24 was mainly located in the membrane and/or cytoplasm of neurons, astrocytes, and microglia. As compared to that of the sham group, the number of CD24positive cells was significantly higher in the TBI group, especially in the neurons and astrocytes as well as the microglia. Bar $=20 \mu \mathrm{m}$ 